\title{
Implementation of Interrogation Systems for Fiber Bragg Grating Sensors
}

\author{
Benjamin VARGHESE P., Dinesh KUMAR R., Mittu RAJU, and \\ K. N. MADHUSOODANAN \\ Department of Instrumentation, Cochin University of Science and Technology, Cochin, India \\ ${ }^{*}$ Corresponding author: Benjamin VARGHESE P. $\quad$ E-mail: benjamin@cusat.ac.in
}

\begin{abstract}
The development of two simple methods for wavelength-optical intensity modulation techniques for fiber Bragg grating (FBG) sensors is presented. The performance is evaluated by measuring the strain and temperature. The first method consists of a narrow band source, an optical circulator, an FBG, and a power meter. The source and Bragg reflected signal from the FBG need to be matched to get linear results with good power levels. The source spectral power levels are very critical in this study. The power reflected from a matched reference FBG is fed into the measuring FBG in the second method. Since the FBGs are matched, the entire power is reflected back initially. During the measurement, the change in the measurand causes the reflected power from the sensing FBG to vary. A costly high resolution spectrum analyzer is required only during the characterization of the FBG and source. The performances of two interrogators are compared by measuring the strain and temperature. In the second method, the strain measurements can be made insensitive to the temperature variation by selecting a source with a flat spectrum at the measurement range. Highlights of these methods are the portability, cost effectiveness and better resolution.
\end{abstract}

Keywords: Fiber Bragg grating, interrogator, reflected power, strain and temperature measurement

Citation: Benjamin VARGHESE P., Dinesh KUMAR R., Mittu RAJU, and K. N. MADHUSOODANAN, "Implementation of Interrogation Systems for Fiber Bragg Grating Sensors," Photonic Sensors, DOI: 10.1007/s13320-013-0108-5.

\section{Introduction}

The fiber Bragg grating (FBG) has proved its potential for measuring the strain, temperature, pressure, displacement, refractive index, humidity, etc. [1-8]. FBGs also find applications in the structural health monitoring as well in the medical field [9-11]. The small size, real-time measurement, fast response, immunity to electromagnetic waves, are the advantages of general fiber optic sensors [12]. Among the fiber optic sensors, FBG sensors have advantages, such as high multiplexing capabilities, distributed sensing, a well localized sensing region and elimination of the frequent calibration [13-15]. In case of spectral measurement, it is insensitive to source fluctuations. The main bottle neck in the use of FBG sensors for different applications is the costly demodulation technique. Even the costly spectrum analyzer can detect up to 4 pico-meter, and the resolution in the strain measurement is approximately $20 \mu \varepsilon$. The dynamic response of the measurement is also poor since every time the spectral components need to be analyzed. In the literature, many optical wavelength-intensity demodulation techniques based on the long period grating, FBG filter, chirped fiber grating, etc., were

Received: 28 December 2012 / Revised version: 9 April 2013

(C) The Author(s) 2013. This article is published with open access at Springerlink.com 
discussed [16-18]. In this study, we suggest two simple methods for the wavelength to intensity conversion.

The first method is by selecting a narrow band source and an FBG with the Bragg reflection at the trailing edge of the source spectrum. So as the Bragg reflected wavelength shifts due to the strain or temperature, the reflected power varies depending on the selection of the Bragg wavelength. A few components are used hence the cost of measurement is less with this method. The main limitation in this study is that the emission of the source and the FBG reflection need to be critically matched. For the second method, two matched FBGs are used. The Bragg reflected signal from the reference FBG is fed into the FBG in which the measurements are carried out. During the measurement, the reflected power varies as the Bragg reflected wavelength shifts. This can be monitored using a power meter. The dynamic response of the measurement will be better, since the intensity is measured. If the source used is a broad band source with a flat spectrum then the impact due to temperature variations will not affect the strain measurements in the second arrangement.

\section{Theory}

According to the Bragg's law, we have

$$
\lambda_{B}=2 n_{\text {eff }} \Lambda
$$

where $\lambda_{B}$ is the Bragg wavelength, $n_{\mathrm{eff}}$ is the refractive index of the fiber core, and $\Lambda$ is the Bragg grating period [19]. The strain measurement is based on the physical elongation of the optical fiber which corresponds to the change in the grating pitch and refractive index of the fiber due to the photo elastic effect. At the constant temperature, the shift in the Bragg wavelength can be expressed as

$$
\Delta \lambda_{B}=\left(1-p_{\varepsilon}\right) \lambda_{B} \varepsilon
$$

where $\varepsilon$ is the applied strain, and $p_{e}$ is the effective photo elastic coefficient, which is expressed as

$$
p_{\varepsilon}=\left(\frac{n^{2}}{2}\right)\left[P_{12}-v\left(P_{11}+P_{12}\right)\right]
$$

where $P_{i j}$ is the Pockel's coefficients of the strain optic tensor, and $v$ is the Poisson's ratio of the optical fiber.

The temperature dependence of the refractive index of silica and thermal expansion of the glass accounts for the Bragg wavelength shift due to the temperature. The major contribution is due to the temperature dependence of the refractive index of silica. The shift in the Bragg wavelength can be expressed as $[20]$

$$
\Delta \lambda_{B}=\left[\frac{1}{\Lambda}\left(\frac{\delta \Lambda}{\delta T}\right)+\frac{1}{n}\left(\frac{\delta n}{\delta T}\right)\right] \lambda_{B} \Delta T .
$$

The first term relates to the thermal expansion of the fiber, and the second term relates to the temperature dependence of the refractive index.

The reflected spectrum of the measuring FBG in the first method is given by [21]

$$
R_{r 1}(\lambda)=R_{r 1} \exp \left[-4 \ln 2\left(\frac{\lambda-\lambda_{r 1}}{\sigma_{r 1}}\right)^{2}\right]
$$

where $R_{r 1}$ is the reflectivity, $\sigma_{r 1}$ is the full width at the half maximum (FWHM) of the FBG. The intensity at the power meter is given by

$$
\int_{0}^{\infty} L(\lambda) R_{r l}(\lambda) d \lambda
$$

where $L(\lambda)$ is the source spectrum, which is expressed as

$$
L(\lambda)=P \exp \left(\frac{-\left(\lambda-\lambda_{p}\right)^{2}}{0.362 \sigma_{s}^{2}}\right)
$$

where $P$ is the peak power, $\lambda_{p}$ is peak emission wavelength, $\sigma_{s}$ is the FWHM of the source. Hence in the first study, the power reaching at the detector is given by (6).

In the second study, this power is given to the measuring FBG. Since the FBGs are matched, this signal is again reflected by the second FBG. The reflected spectrum is given by

$$
R_{r 2}(\lambda)=R_{r 2} \exp \left[-4 \ln 2\left(\frac{\lambda-\left(\lambda_{r 2}+\Delta \lambda\right.}{\sigma_{r 2}}\right)^{2}\right]
$$

where $\lambda_{r 2}=\lambda_{r 1}+\Delta \lambda_{x}$ with $\Delta \lambda_{x}$ accountable for the slight mismatch between two FBGs, and $\Delta \lambda$ is the shift in the Bragg wavelength due to the applied strain. Initially for matched FBGs, 


$$
R_{r 1}=R_{r 2}, \sigma_{r 1}=\sigma_{r 2} .
$$

Hence without the strain in the second FBG, $\Delta \lambda$ is zero and

$$
R_{r 1}(\lambda) \approx R_{r 2}(\lambda)
$$

Intensity $I$ is given by

$$
\int_{0}^{\infty} L(\lambda) R_{r 1}(\lambda) R_{r 2}(\lambda) d \lambda .
$$

On substitution, the reflected power at the power meter is given by

$$
\begin{gathered}
\int_{0}^{\infty} L(\lambda) R_{r 1} R_{r 2} \exp \left[-4 \ln 2\left\{\left(\frac{\lambda-\lambda_{r 1}}{\sigma_{r 1}}\right)^{2}+\right.\right. \\
\left.\left(\frac{\lambda-\left(\lambda_{r 2}+\Delta \lambda\right)}{\sigma_{r 2}}\right)^{2}\right\} .
\end{gathered}
$$

\section{Experimental details}

The experimental setup shown in Fig. 1 has a laser source [ILX Light wave 7900B], an optical circulator having 3 ports, an FBG pasted to a cantilever setup and a power meter. The laser source has an emission peak at $1550 \mathrm{~nm}$. The FBG has a Bragg wavelength of $1550 \mathrm{~nm}$ with a grating length of $13 \mathrm{~mm}$, and the reflectivity offered is $90 \%$. The laser source is connected to the port 1 of the circulator. In the port 2, the FBG pasted to the cantilever structure is connected. A power meter (Hewlett-Packard, 8153A) is connected at the port 3 of the circulator to measure the Bragg reflected power. The cantilever for the strain measurement has the length of $20 \mathrm{~cm}$, breadth of $5 \mathrm{~cm}$ and is made in a spring steel of 2-mm thickness. Using a fast setting epoxy, the FBG is pasted to the cantilever structure. The strain is applied in the range of $0-1000 \mu \varepsilon$, and it is monitored using electrical strain gauges. The reflected spectrum is measured for every $100 \mu \varepsilon$ increment. A vibration free table has been used, and the sufficient time is allowed to avoid loading transients.

In the second setup shown in Fig. 2, the first port of the optical circulator is connected to the laser source, and the second port is connected to the reference FBG, which is matched with the measuring FBG. The third port is connected to the $3-\mathrm{dB}$ coupler. The FBG is pasted to the cantilever structure for the strain measurement, and the power meter is connected as shown in Fig.2. The reflected signal from the strain measuring setup is monitored using an optical spectrum analyzer (OSA) [Yokogawa, AQ 6319], as well as the power meter.

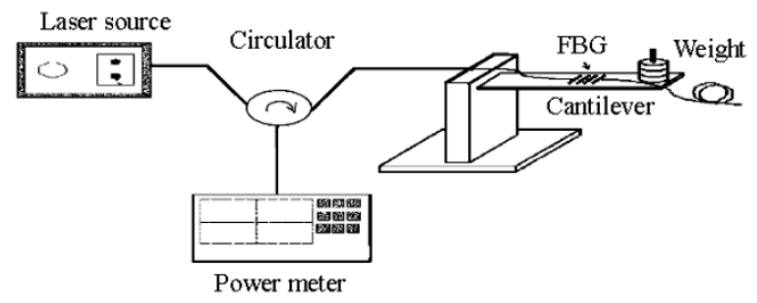

Fig. 1 Experimental setup for the strain measurement.

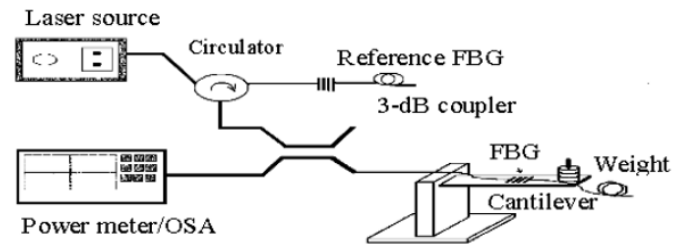

Fig. 2 Experimental setup for the strain measurement with the reference FBG.

To measure the temperature, the FBG is immersed in the water bath, and the temperature varies from $22^{\circ} \mathrm{C}$ to $100^{\circ} \mathrm{C}$. A digital thermometer is also used for monitoring the temperature of the water bath. The reflected spectrum is monitored for a temperature rise of $10{ }^{\circ} \mathrm{C}$. Hysterises of the temperature measurement is monitored as the water bath cools down naturally.

\section{Results and discussion}

The source spectrum is monitored, which is depicted in Fig. 3. The emission peak is observed at $1550 \mathrm{~nm}$, and then the power level drops linearly and

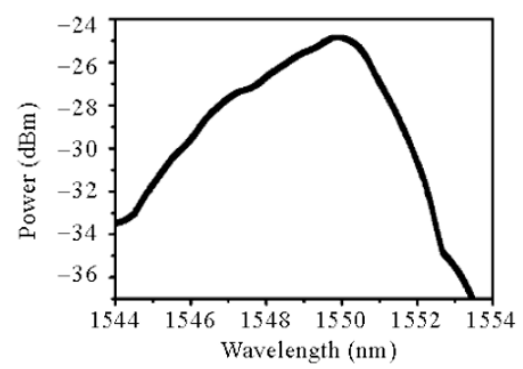

Fig. 3 Source spectrum. 
makes it ideal for the measurement without a reference FBG. The spectral stability of the source is very critical in this study. The source used is very stable, and the temperature maintains at $22{ }^{\circ} \mathrm{C}$.

The reflected spectrum at the port 3 is simulated using the MATLAB tool for a laser diode of the emission peak at $1550 \mathrm{~nm}$ for 0 to $1000 \mu \varepsilon$ in increments of $200 \mu \varepsilon$, as shown in Fig.4(a). The port 3 of the circulator is monitored using the OSA, depicted in Fig. 4(b). The peak power level is ranging from $-30 \mathrm{dBm}$ to $-60 \mathrm{dBm}$ for a strain of $0-1000 \mu \varepsilon$. The power variation is almost linear in this range. The FBG Bragg reflection is at the trailing edge of the source spectrum, and as the Bragg reflected wavelength red shifts due to the strain or temperature, the reflected power decreases. In the reflected spectrum observed in the OSA, the leading edge shows a red shift in the wavelength due to the strain. However, there is no red shift at the trailing edge as the response of the source does not cover this wavelength range. The selection of the Bragg wavelength of the FBG and the source emission peak is very critical in this configuration.

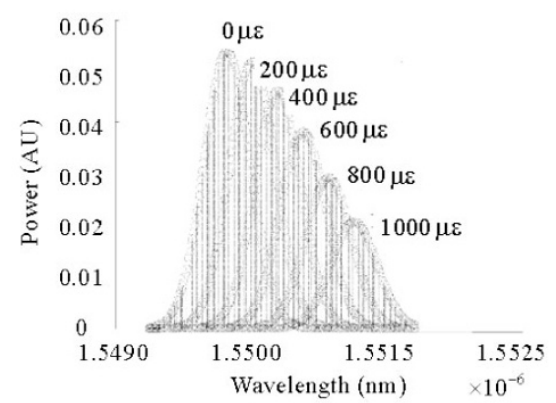

(a)

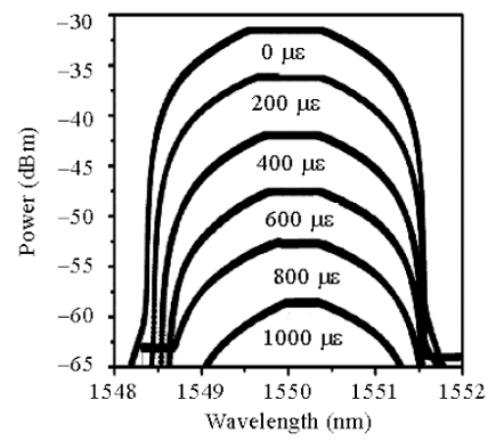

(b)

Fig. 4 Reflected spectrum during strain measurement without the reference FBG: (a) simulated and (b) measured.
In the second setup, a reference FBG is used in addition to the sensing FBG. The reflected signal from the reference FBG is sourced to the strain sensing FBG. The reflected spectrum from the sensing FBG is measured for 0 to $1000 \mu \varepsilon$. The simulated results for strain variation from 0 to $1000 \mu \varepsilon$ in increments of $200 \mu \varepsilon$ are shown in Fig. 5(a). The measured reflected spectrum from 0 to $1000 \mu \varepsilon$ is shown in Fig. 5(b). Since the FBGs are matched, the same reflected spectral components from the reference FBG are reflected by the sensing FBG initially without the strain. It is observed that as the strain increases, the reflected spectrum red shifts, and the power reflected from the second FBG decreases. In the simulated figure, the results up to $600 \mu \varepsilon$ are only visible since the power levels for $800 \mu \varepsilon$ and $1000 \mu \varepsilon$ are very near to the base line. The red shift of the reflected spectrum is shown clearly in the simulated results where as in the actual output from the sensing FBG only the leading edge shows the red shift. The trailing edge is not showing the shift because the spectral width of the source does not extent to the higher wavelength.

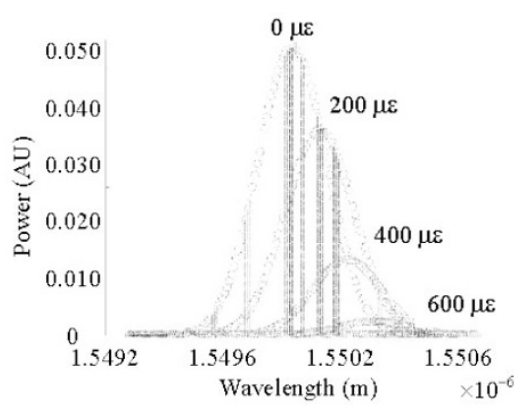

(a)

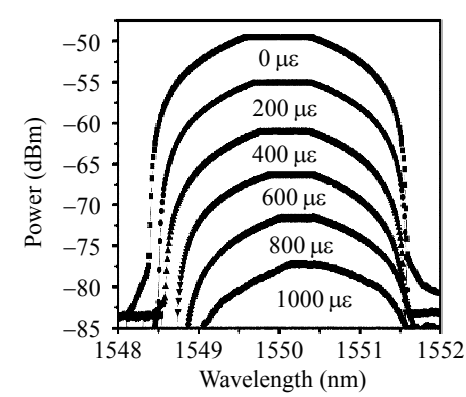

(b)

Fig. 5 Reflected spectrum during strain measurement with the reference FBG: (a) simulated and (b) measured. 
The reflected power with and without the reference is shown in Fig. 6. In the measurement without the reference, the power varies from $-20.33 \mathrm{dBm}$ to $-41.21 \mathrm{dBm}$ for a strain variation of 0 to $1000 \mu \varepsilon$. Whereas the reflected power varies linearly from $-37.39 \mathrm{dBm}$ to $-65.21 \mathrm{dBm}$ with the reference for the same range. The performances of the FBGs are evaluated using a broad band source. The leading edge of the reflected spectrum shifts from $1549.8 \mathrm{~nm}$ to $1550.8 \mathrm{~nm}$ linearly for the strain range of 0 to $1000 \mu \varepsilon$. Analyzing the spectral components of the reflected spectrum is time consuming, and the dynamic response of the measurement is poor. The suggested interrogation system actually measures the intensity hence the measurement will have better dynamic response.

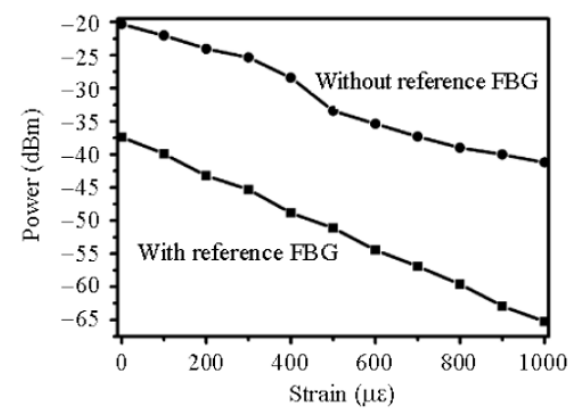

Fig. 6 Power variation during strain measurement.

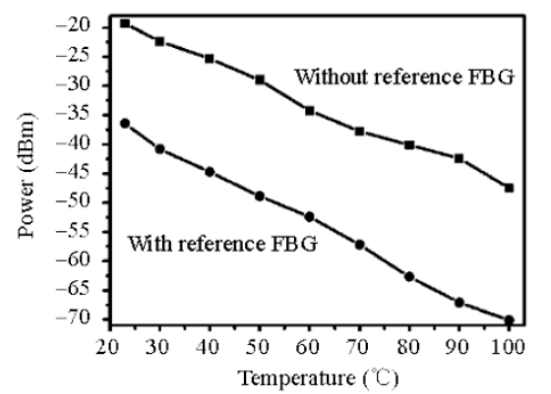

Fig. 7 Power variation for the temperature from $220{ }^{\circ} \mathrm{C}$ to $100{ }^{\circ} \mathrm{C}$.

The second arrangement can be made insensitive to the temperature variation during the strain measurement using a broad band source. As the temperature shifts, the reflected spectrum from both the FBGs will shift evenly. But a decrease in the power is only due to the applied strain in the second FBG. Hence, the strain measurement can be made insensitive to the temperature variation.

The temperature is measured from $22{ }^{\circ} \mathrm{C}$ to $100{ }^{\circ} \mathrm{C}$. The reflected power variations for the temperature measurement with and without the reference FBG are plotted in Fig. 7.

\section{Conclusions}

Two interrogation methods for FBG sensors are developed, and their performances are compared. If the emission spectra of the source and FBG are matched perfectly, the first method is appropriate, since it can provide better power levels with less number of components. But the stability and spectral response of the source are critical. In the second design, the source response is not very critical as in the earlier case. This method is appropriate, and linear results are obtained. It can be made temperature insensitive by using a powerful broadband source with a flat spectrum in the measurement range. Using a 4 pico-meter resolution OSA, we can measure a minimum strain of $24 \mu \varepsilon$ and temperature of $0.1{ }^{\circ} \mathrm{C}$. Whereas using this arrangement, the measurement up to $0.5 \mu \varepsilon$ and $0.01{ }^{\circ} \mathrm{C}$ is possible with a power meter of $0.01 \mathrm{dBm}$ resolution, provided the source power is constant. The highlights of this design are the portability, cost effectiveness and better resolution. Since the intensity is being measured, the dynamic response also expects better.

\section{Acknowledgement}

The authors are thankful to Nest Optoelectronics division for providing necessary assistance during measurement. B. V. P is thankful to University Grants Commission, Government of India for providing the research fellowship during the course of this work.

Open Access This article is distributed under the terms of the Creative Commons Attribution License which permits any use, distribution, and reproduction in any medium, provided the original author(s) and source are 
credited.

\section{References}

[1] E. Udd, C. Lawrence, and D. Nelson, "Development of a three axis strain and temperature fibre optic grating sensor," in Proc. SPIE, vol. 3042, pp. 229-236,1997.

[2] C. H. Lee, M. K. Kim, K. T. Kim, and J. Lee, "Enhanced temperature sensitivity of fiber Bragg grating temperature sensor using thermal expansion of copper tube," Microwave and Optical Technology Letters, vol. 53, no. 7, pp. 1669-1671, 2011.

[3] A. Kersey, M. A. Davis, H. J. Patrick, M. Leblanc, K. P. Koo, C. G. Askins, et al.,"Fiber grating sensors," Journal of Lightwave Technology, vol. 15, no. 8, pp. 1442-1463, 1997.

[4] X. Shu, L. Zhang, and I. Bennion, "Sensitivity characteristics of long-period fiber gratings," Journal of Lightwave Technology, vol. 20, no. 2, pp. 255-266, 2002.

[5] M. G. Xu, L. Reekie, Y. T. Chow, and J. P. Dakin, "Optical in-fiber grating high pressure sensor," Electronics Letters, vol. 29, no. 4, pp. 398-399,1993.

[6] I Yong Zhao, He Huang, and Qi Wang, "Interrogation technique using a novel spectra bandwidth measurement method with a blazed FBG and a fiber-optic array for an FBG displacement sensor," Sensors Actuators A: Physical, vol. 165, no. 2, pp. 185-188, 2011.

[7] W. Liang, Y. Huang, Y. Xu, R. K. Lee, and A. Yariv, "Highly sensitive fiber Bragg grating refractive index sensors," Applied Physics Letters, vol. 86, no. 15, pp. 151122-1-151122-3, 2005.

[8] T. L. Yeo, T. Sun, K. T. V. Grattan, D. Parry, R. Lade, and B. D. Powell, "Characterization of a polymer-coated fiber Bragg grating sensor for relative humidity sensing," Sensors and Actuators B: Chemical, vol. 110, no. 1, pp.148-155, 2005.

[9] R. M. Measures, "Smart composite structures with embedded sensors," Composites Engineering, vol. 2, no. 5-7, pp. 597-618, 1992.

[10] G. Zhou and L. M. Sim, "Damage detection and assessment in fiber-reinforced composite structures with embedded fiber optic sensors-review," Smart Materials and Structures, vol. 11, no. 6, pp. 925-939, 2002.

[11] A. F. Silva, J. P. Carmo, P. M. Mendes, and J. H. Correia, "Simultaneous cardiac and respiratory frequency measurement based on a single fiber Bragg grating sensor," Measurement Science and Technology, vol. 22, no. 22, pp. 075801, 2011.

[12] S. Yin, P. B. Ruffin, and F. T. S. Yu, Fiber optic sensor. England: Press Taylor \& Francis Group, 2008.

[13] S. Kutaran, and M. S. Kilickaya, "The modeling of fibre Bragg grating," Optical and Quantum Electronics, vol. 39, no. 8, pp. 643-650, 2007.

[14] H. N. Li, D. S. Li, and G. B. Song, "Recent applications of fiber optic sensors to health monitoring in civil engineering," Engineering Structures, vol. 26, no. 11, pp. 1647-1657, 2004.

[15] M. Majumder, T. K. Gangopaadhyay, A. K. Chakraborty, K. Dasgupta, and D. K. Bhattacharya, "Fiber Bragg gratings in structural health monitoring - present status and applications," Sensors and Actuators A: Physical, vol. 147, no. 1, pp. 150-164, 2008.

[16] R. W. Fallon, L. Zhang, L. A. Everall, J. A. R. Williams, and I. Bennion, "All-fiber optical sensing system: Bragg grating sensor interrogated by a long period grating," Measurement Science and Technology, vol. 9, no. 12, pp. 1969-1973, 1998.

[17] I. C. Song, S. K. Lee, S. H. Jeong, and B. H. Lee, "Absolute strain measurement made with fiber Bragg grating sensors," Applied Optics, vol. 43, no. 6, pp. 1337-1341, 2004.

[18] S. Kim, S. Kim, J. Kwon, and B. Lee, "Fiber Bragg grating strain sensor demodulator using a chirped fiber grating," IEEE Photonics Technology Letters, vol. 13, no. 8, pp. 839-841, 2001.

[19] R. Kashyap, Fibre Bragg grating. London: Academic Press, 1999.

[20] B. D. Gupta, Fibre optic sensors principles and applications. New Delhi: New India Publications, 2006.

[21] K. C. Chuang and C. C. Ma, "Multidimensional dynamic displacementandstrain measurement using an intensity demodulation-based fiber-Bragg grating sensing system," Journal of Lightwave Technology, vol. 28, no. 13, pp. 1897-1905, 2010. 